\title{
P 216 ANALYSIS OF DIAMORPHINE DOSES PRESCRIBED FOR PATIENTS RECEIVING 'JUST IN CASE FOUR CORE DRUGS' FOR END OF LIFE CARE
}

Andrew Dickman, ${ }^{1}$ Grace Khoo, ${ }^{1}$ Tracey Morris ${ }^{2} .{ }^{1}$ Blackpool Teaching Hospitals NHS Foundation Trust, Blackpool, UK; ${ }^{2}$ Trinity Hospice, Blackpool

10.1136/bmjspcare-2014-000654.257

Background In September 2012, an anticipatory prescribing policy was introduced across the healthcare. The 'Just in Case Four Core Drugs' (JiC4CD) policy can support proactive symptom control by promoting anticipatory prescribing, ensuring that common symptoms (for example nausea and vomiting, pain, respiratory secretions and agitation) are responded to in a timely fashion.

The four core drugs have been chosen for the locality because they are consistent with the prescribing algorithms of the local Liverpool Care Pathway. These drugs are diamorphine, glycopyrronium, levomepromazine and midazolam, although alternatives are acceptable should they be necessary (e.g. oxycodone instead of diamorphine). A recent national survey concluded that morphine, not diamorphine, is the most commonly prescribed opioid for administration by continuous subcutaneous infusion. There is a potential cost saving by substituting morphine for diamorphine.

Aims To analyse the doses of diamorphine prescribed for patients receiving $\mathrm{JiC} 4 \mathrm{CD}$ and to determine the feasibility of switching to morphine.

Method Electronic discharge prescriptions over a 12-month period for JiC4CD were identified. Those prescriptions for diamorphine were analysed to determine the mean, median and mode doses prescribed.

Results A total of 211 discharge prescriptions for JiC4CD were dispensed by the hospital pharmacy over the 12 month period. There were 193 (91.4\%) discharge prescriptions that included diamorphine as the opioid of choice. The mean $(4.77 \mathrm{mg})$, median $(5 \mathrm{mg})$ and mode $(5 \mathrm{mg})$ doses were determined.

Conclusion The analysis illustrated that morphine could be considered as an alternative to diamorphine for patients receiving JiC4CD for end-of-life care. 